Recepción: 05 / 09 / 2018

Aceptación: 08 / 11 / 2018

Publicación: 03 / 12 / 2018

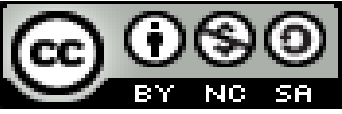

Ciencias técnicas y aplicadas

Artículo original

\title{
Análisis experimental de la fuerza de arrastre en automóviles modelo por el flujo de fluido
}

\section{Experimental analysis of drag force by fluid flow in model cars}

\section{Análise experimental de força de arrasto por fluxo de fluido em carros modelo}

\author{
Lenin S. Orozco-Cantos ${ }^{\mathrm{I}}$ \\ 1sorozco@espoch.edu.ec \\ Santiago A. López-Ortiz II \\ sa_lopez@ espoch.edu.ec \\ John G. Vera-Luzuriaga III \\ John.vera@espoch.edu.ec \\ Marco A. Ordoñez-Viñán ${ }^{\mathrm{IV}}$ \\ marco.ordonez@espoch.edu.ec
}

Correspondencia: 1sorozco@espoch.edu.ec

\footnotetext{
I Magíster en Eficiencia Energética, Ingeniero Mecánico, Docente de la Escuela Politécnica de Chimborazo ESPOCH, Riobamba, Chimborazo, Ecuador.

II Magíster en Diseño Mecánico, Ingeniero Mecánico, Docente de la Escuela Politécnica de Chimborazo ESPOCH, Riobamba, Chimborazo, Ecuador.

III Máster Universitario en Ingeniería Mecatrónica, Ingeniero en Electrónica, Control y Redes Industriales, Docente de la Escuela Politécnica de Chimborazo ESPOCH, Riobamba, Chimborazo, Ecuador.

IV Magíster en Eficiencia Energética, Ingeniero Mecánico, Docente de la Escuela Politécnica de Chimborazo ESPOCH, Riobamba, Chimborazo, Ecuador.
} 


\section{Resumen}

El movimiento de cuerpos en el contexto aerodinámico presenta un factor muy importante, el cual se conoce como coeficiente de arrastre, este coeficiente presenta un valor respecto del cual se puede interpretar si la geometría de dicho cuerpo tiene una menor o mayor resistencia al movimiento debido a la fuerza que se opone a dicho movimiento conocida como fuerza de arrastre. Los automóviles comerciales o de uso urbano evidentemente presentan una valoración de este coeficiente, mismo que en muy pocos casos se cuenta con esta información fácilmente, si un usuario pretende conocer a detalle la información de su vehículo, con el objetivo de prever si la eficiencia en el consumo de combustible tiene factores residuales que podrían aportar a la misma, se hace necesario identificar el valor del coeficiente de arrastre de dicho vehículo, puesto que mientras menor sea este, el consumo innecesario de combustible no será por la geometría o diseño exterior del mismo. Atendiendo esta premisa, este coeficiente se lo puede determinar a través de experimentación en un banco de flujo externo ajustable utilizando modelos a escala de los cuerpos de interés, en el caso de estudio se ha utilizado los modelos de Citroen GS, Alfa Romeo 159, Fiat 128; de los cuales el fabricante provee la información completa y se puede cotejar los datos experimentales con los teóricos, obteniéndose en esta comparativa valores similares, el grado de confiablidad del banco es alto por lo cual se podría replicar este tipo de experimentaciones para cualquier otro modelo de vehículo.

Palabras clave: coeficiente de arrastre; aerodinámico; experimentación en banco de flujo externo; aerodinámico.

\section{Abstract}

The movement of bodies in the aerodynamic context presents a very important factor, which is known as drag coefficient, this coefficient presents a value with respect to which it can be interpreted if the geometry of said body has a lower or greater resistance to movement due to the force that opposes this movement known as drag force. Commercial or urban vehicles evidently present an assessment of this coefficient, even though in very few cases this information is easily available, if a user pretends to know in detail the information of his vehicle, in order to predict whether the efficiency in the fuel consumption has residual factors that could contribute to it, it is necessary to identify the value of the drag coefficient of said vehicle, since the lower it is, the unnecessary fuel consumption will not be due to the geometry or exterior design thereof. Taking 
this premise into account, this coefficient can be determined through experimentation in an adjustable external flow bank using scale models of the bodies of interest, in the case of the study it has been used the models of Citroen GS, Alfa Romeo 159, Fiat 128; of which the manufacturer provides the complete information and can compare the experimental data with the theoretical, obtaining in this comparative similar values, the degree of reliability of the bank is high which could replicate this type of experimentation for any other model of vehicle.

Keywords: Drag coefficient; aerodynamic; experimentation in bank of external flow; aerodynamic.

\section{Resumo}

O movimento de organismos no ambiente aerodinâmico apresenta um factor muito importante, que é conhecido como o coeficiente de arrasto, este coeficiente é um valor para o qual pode ser interpretada se a geometria do referido corpo tem um menor ou maior resistência ao movimento, devido à a força que se opõe a esse movimento, conhecida como força de arrasto. carros de uso comercial ou urbanas, obviamente, têm uma avaliação deste coeficiente, como em muito poucos casos têm esta informação facilmente, se um usuário tenta conhecer em detalhe as informações em seu veículo, a fim de prever se a eficiência consumo de combustível tem factores residuais que poderiam contribuir para isso, é necessário identificar o valor do coeficiente de arrasto do veículo, uma vez que o menor isso, o consumo desnecessário de combustível não será pela geometria ou desenho exterior da mesma . Em resposta a esta premissa, esta proporção seria determinada através de experimentação de um banco de fluxo externo ajustável usando modelos em escala de organismos de interesse no estudo de caso modelos utilizados Citroen GS, Alfa Romeo 159, Fiat 128; de que o fabricante proporciona informação completa e pode comparar os dados experimentais com o rendimento teórico nesta valores semelhantes de comparação, o grau de banco dirigibilidade é tão alta poderia replicar este tipo de experimentações para qualquer outro modelo veiculo.

Palavras chave: coeficiente de arrasto; aerodinâmico; experimentação em banco de fluxo externo; aerodinâmico. 


\section{Introducción}

Todo cuerpo que esté inmerso en la corriente de un fluido estará sometido a fuerzas y momentos de fuerzas que dependen de la forma y orientación con respecto al flujo. La fuerza paralela al flujo se llama arrastre o resistencia al aire. Este arrastre tiene signo positivo cuando va en el sentido del flujo. Si un objeto ha de moverse contra el flujo deberá vencer a esta fuerza. Es importante entender que estas fuerzas son definiciones prácticas que representan el efecto de los rebotes y el deslizamiento de las partículas contra la superficie del cuerpo. (1)

$\mathrm{El}$ arrastre es una fuerza mecánica. Es generada por la interacción y contacto de un cuerpo rígido y un fluido. No es generado por un campo de fuerzas como en el caso de fuerzas gravitacionales o electromagnéticas donde no es necesario el contacto físico. Para que exista arrastre el cuerpo debe estar en contacto con el fluido. Debe haber un movimiento relativo entre el fluido y el sólido. (2)

Siendo una fuerza, el arrastre es un vector que va en la dirección contraria al movimiento del cuerpo. Existen muchos factores que afectan la magnitud del arrastre. La magnitud de la sección efectiva de impacto y la forma de la superficie. Un efecto que produce arrastre es el de roce aerodinámico con la superficie llamado efecto piel entre las moléculas del aire y las de la superficie sólida. Una superficie muy suave y encerada produce menos arrastre por este efecto que una rugosa. A su vez este efecto depende de la magnitud de las fuerzas viscosas. A lo largo de la superficie se genera una capa de borde formada por moléculas de baja energía cinética y la magnitud de la fricción de piel depende de las características de esta capa. Se encuentra en la vecindad inmediata de la superficie del cuerpo. (3)

Otro efecto muy importante es el de arrastre de forma, la forma de un cuerpo produce una determinada distribución de las presiones debido a las velocidades locales. Integrando estas presiones sobre toda la superficie del cuerpo obtendremos la fuerza de arrastre. Existen otros tipos de arrastre llamados arrastres inducidos que son producidos por la dinámica del flujo debido a la forma particular del cuerpo. Los vórtices que se producen en las puntas de las alas de los aviones generan este tipo de arrastre. Las alas muy cortas y anchas tienen grandes arrastres. La formación de ondas de choque al acercarse un cuerpo a la velocidad del sonido en el fluido es fuente también de resistencia al movimiento. (1) 
Se conoce que la aerodinámica es la parte de la mecánica de fluidos que se encarga de estudiar los fenómenos que se originan cuando existe movimiento relativo entre un sólido y el fluido gaseoso que lo rodea, determinando las presiones y fuerzas que se van a generar. La magnitud de las fuerzas aerodinámicas que se generan cuando un vehículo se desplaza por la carretera, va a depender de las características del aire (viscosidad y densidad) y del sólido. El sólido, en este caso un automóvil, ha de considerarse su forma, su rugosidad superficial, el área de contacto con el agua y, sobre todo, la velocidad relativa entre éste y el agua. (4)

En el estudio experimental de las fuerzas de arrastre existe un factor muy importante, el cual es la relación que se consigue asignar entre el modelo y el prototipo d experimentación. Entiéndase que por modelo se refiere al cuerpo construido para experimentación en laboratorio, mientras que prototipo es el cuerpo de dimensiones reales que funcionara en condiciones reales. La similitud incompleta se muestra con el problema de medir la fuerza aerodinámica de arrastre de un modelo de camión en un túnel de viento, por ejemplo. A través del número de Reynolds se puede relacionar las condiciones de experimentación para el modelo y el prototipo, como se indica en la ecuación 1 (5):

$$
R e_{m}=\frac{\rho_{m} V_{m} L_{m}}{\mu_{m}}=R e_{p}=\frac{\rho_{p} V_{p} L_{p}}{\mu_{p}} \quad \text { Ec. } 1
$$

Donde:

Subíndices m: modelo, subíndices p: prototipo

Re: Reynolds

$\rho:$ Densidad

$V:$ Velocidad

$L:$ Longitud característica

$\mu$ : Viscosidad dinámica

En muchas ocasiones es imposible empatar el número de Reynolds del modelo con el del prototipo usando este modelo y el túnel de viento. Entonces existen varias opciones: 
- Si se tuviera un túnel de viento más grande, se podrían hacer pruebas con un modelo más grande. Los fabricantes de automóviles usualmente prueban con modelos de autos a una escala de tres octavos y con modelos de tractocamiones y autobuses a un octavo de escala en túneles de viento muy grandes. Algunos túneles de viento son incluso lo suficientemente grandes como para probar automóviles a tamaño real. Sin embargo, mientras más grandes sean el túnel de viento y el modelo, más costosas serán las pruebas.

- Se podría usar un fluido diferente para las pruebas del modelo. Por ejemplo, los túneles de agua pueden lograr números de Reynolds más altos que los que pueden lograr túneles de viento del mismo tamaño, pero son mucho más costosos de construir y operar.

- Se podría presurizar el túnel de viento y/o ajustar la temperatura del aire para aumentar la capacidad del máximo número de Reynolds. Aunque estas técnicas pueden ayudar, el aumento en el número de Reynolds es limitado.

- Finalmente, se podría correr el túnel de viento a diversas velocidades cercanas a la velocidad máxima, y luego extrapolar los resultados al número de Reynolds a tamaño real.

\section{Metodología}

Para el coeficiente de arrastre se puede utilizar muchos objetos, para la investigación propuesta se ha utilizado vehículos, los cuales en la práctica se somete a pruebas aerodinámicas con aire, pero en este caso se usa un banco de flujo externo, el cual trabaja con agua, debido a las causas antes mencionadas. Entonces un flujo de agua pasa por un ducto rectangular, el caudal es proporcionado por una bomba ubicada en un extremo del banco, el fluido impacta en la sección transversal delantera del vehículo a escala, se genera un perfil de velocidades de acuerdo a la geometría del carro, cada modelo se conecta a un instrumento de medición de fuerza, dinamómetro convencional de resorte, el dinamómetro recorre e indica un valor, que será la fuerza de arrastre generada en el cuerpo de estudio.

En la Figura 1, se muestran los modelos de los vehículos para el análisis. Los mismos fueron construidos a una escala 1:21. Se ha tratado de seleccionar modelos comerciales y que principalmente cuenten con información que provea su fabricante respecto del coeficiente de arrastre, esta información en la mayoría de vehículos es muy difícil de conseguirla. Debido a que 
se pretende comparar los valores de los coeficientes de arrastre, teóricos y experimentales, se presenta a continuación los valores de los mencionados coeficientes (4), véase la Figura 2.

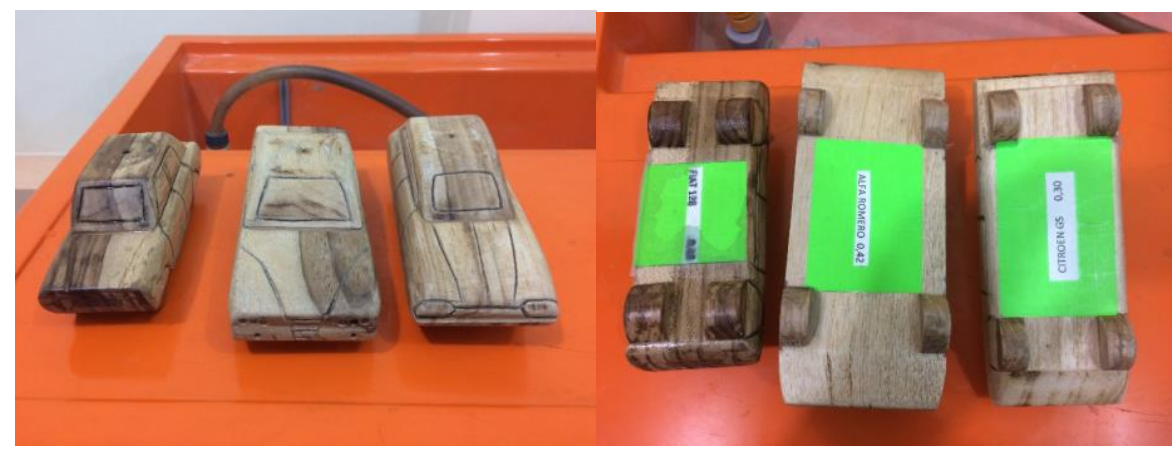

Figura 1. Modelos de autos para la experimentación.

Fuente: Autor
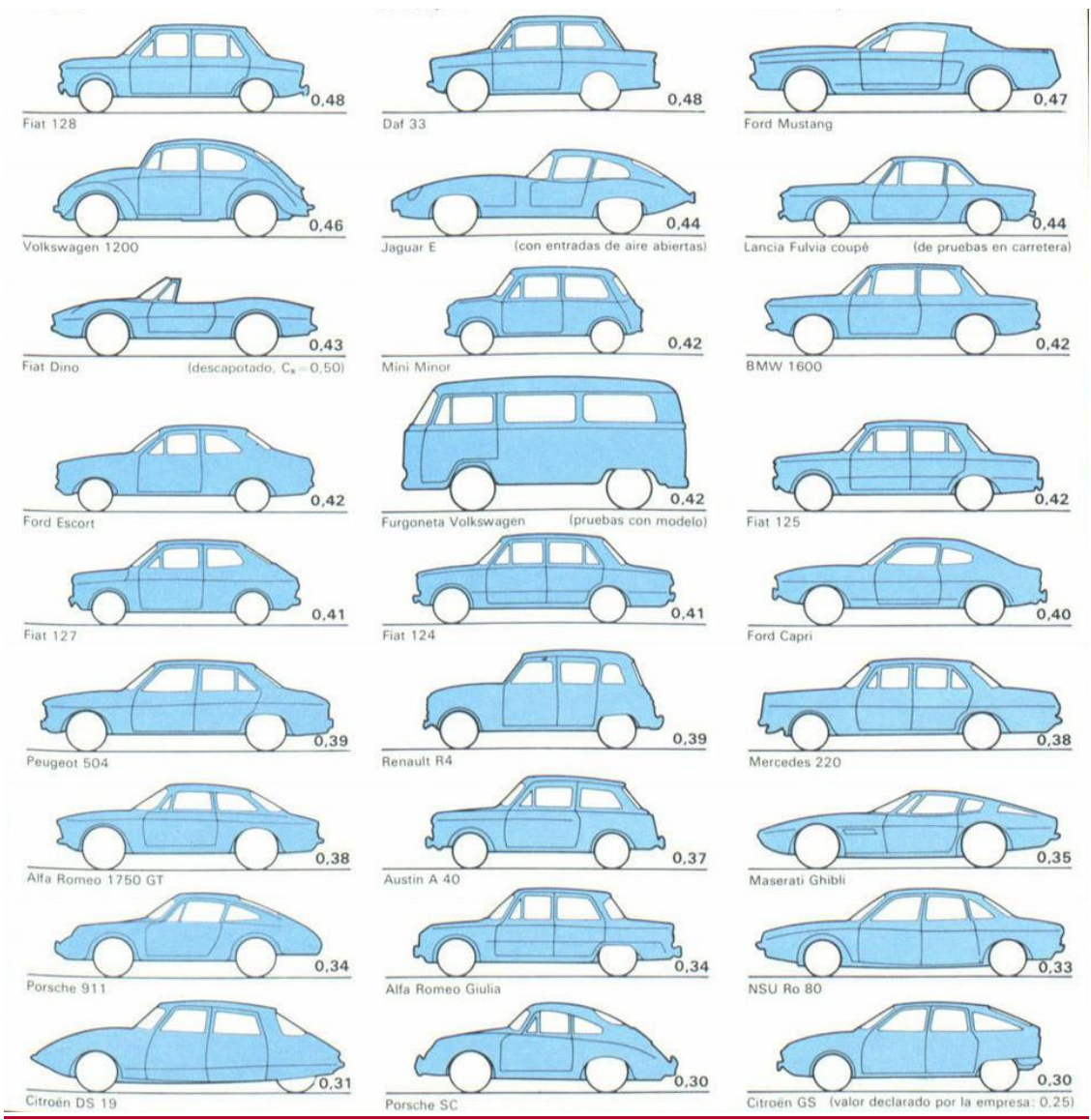

Figura 2. Coeficientes de arrastre teóricos

Fuente: http://www.technicalcourses.net/portal/es/blog/blog_entrada.php?entrada_id=89. 
Son tres los vehículos escogidos, los detalles de los mismos se presenta a continuación:

\section{Vehículo Citroën Gs}

- Constructor: Citroën

- Años de producción: 1970-1981 (GS) / 1979-1986 (GSA)

- Producción total: 1.896.742 unidades (GS) / 576.757 unidades (GSA)

- Modelo precedente: ninguno

- Modelo siguiente: Citroën BX

- Clase: Berlina

- Motores: 1015 a $1299 \mathrm{~cm}^{3}$

- Transmisión: Por tracción

- Longitud: $4200 \mathrm{~mm}$

- Ancho: $1600 \mathrm{~mm}$

- Alto: $1350 \mathrm{~mm}$

- Peso: 900 a $950 \mathrm{~kg}$

- Plataforma común: No existe

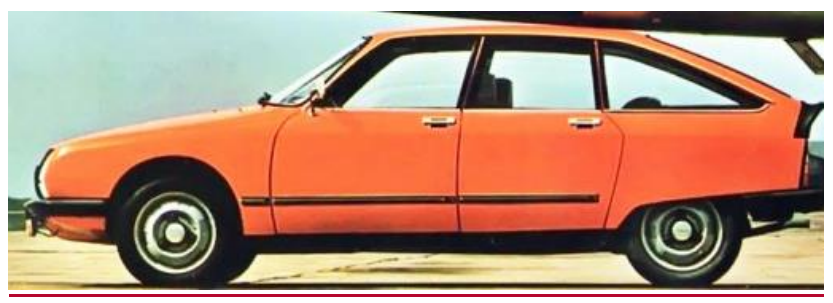

Figura 3 Citroen Gs Coeficiente arrastre 0,30

Fuente: http://www.escuderia.com/citroen-gs-mejor-coche-del-ano-de-la-historia/ 

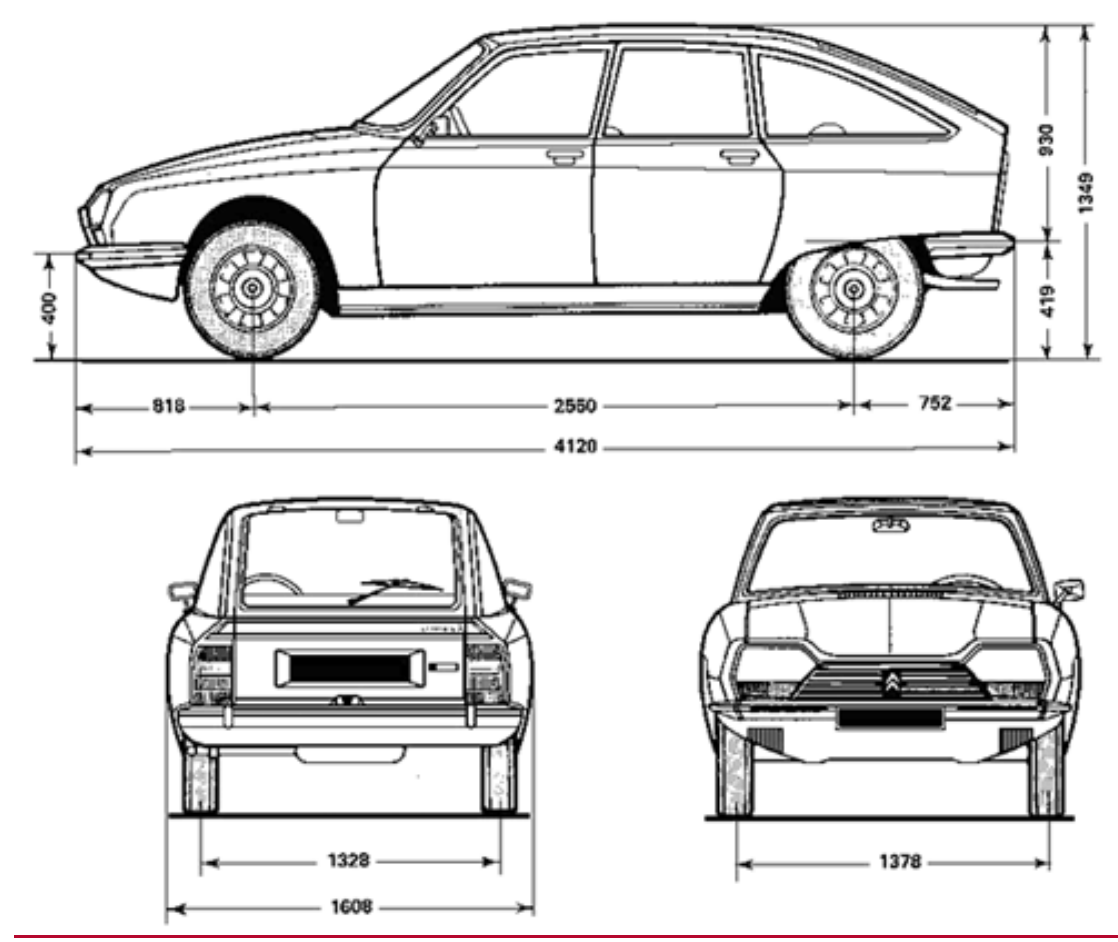

Figura 4 Principales dimensiones Citroen Gs

Fuente: http://www.escuderia.com/citroen-gs-mejor-coche-del-ano-de-la-historia/

\section{Alfa Romeo 159}

- Distribución: 2 ACT accionado mediante correa dentada, taqués hidráulicos

- Número de cilindros: 4 en línea

- Válvulas por cilindro: 4

- Cilindrada (cc): 1910

- Potencia Máxima: 150 Cv / 4.000 r.p.m.

- Diámetro de giro: $11,1 \mathrm{~m}$

- Largo (milímetros): $4.660 \mathrm{~mm}$

- Ancho (milímetros): $1.828 \mathrm{~mm}$

- Alto (milímetros): $1.417 \mathrm{~mm}$

- Distancia entre ejes (mm): $2.700 \mathrm{~mm}$

- Vía delantera (mm): $1.593 \mathrm{~mm}$

- Vía trasera (mm): $1.573 \mathrm{~mm}$

- Peso en orden de marcha: $1.490 \mathrm{~kg}$ 
- Peso máximo de remolque: $1.500 \mathrm{~kg}$

- Capacidad maletero: 405 litros

- Depósito combustible (litros): 70 litros

- Velocidad máxima (km/h): $212 \mathrm{~km} / \mathrm{h}$

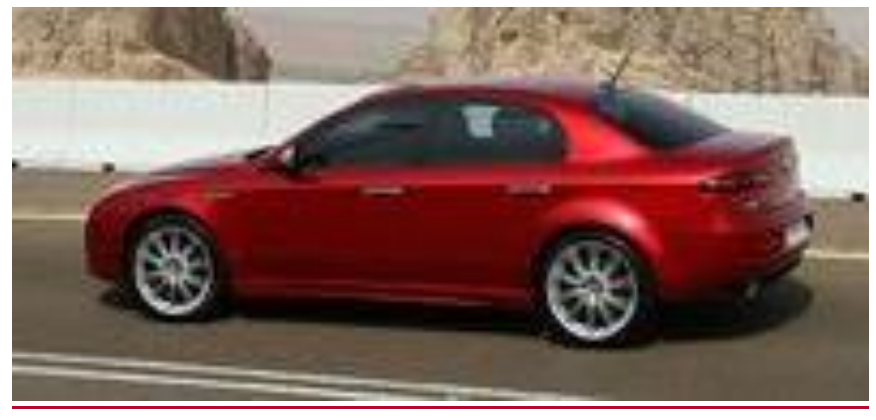

Figura 5 Alfa Romeo 159 Coeficiente de arrastre 0,42

Fuente: https://8000vueltas.com/2010/09/15/citroen-gs-birotor

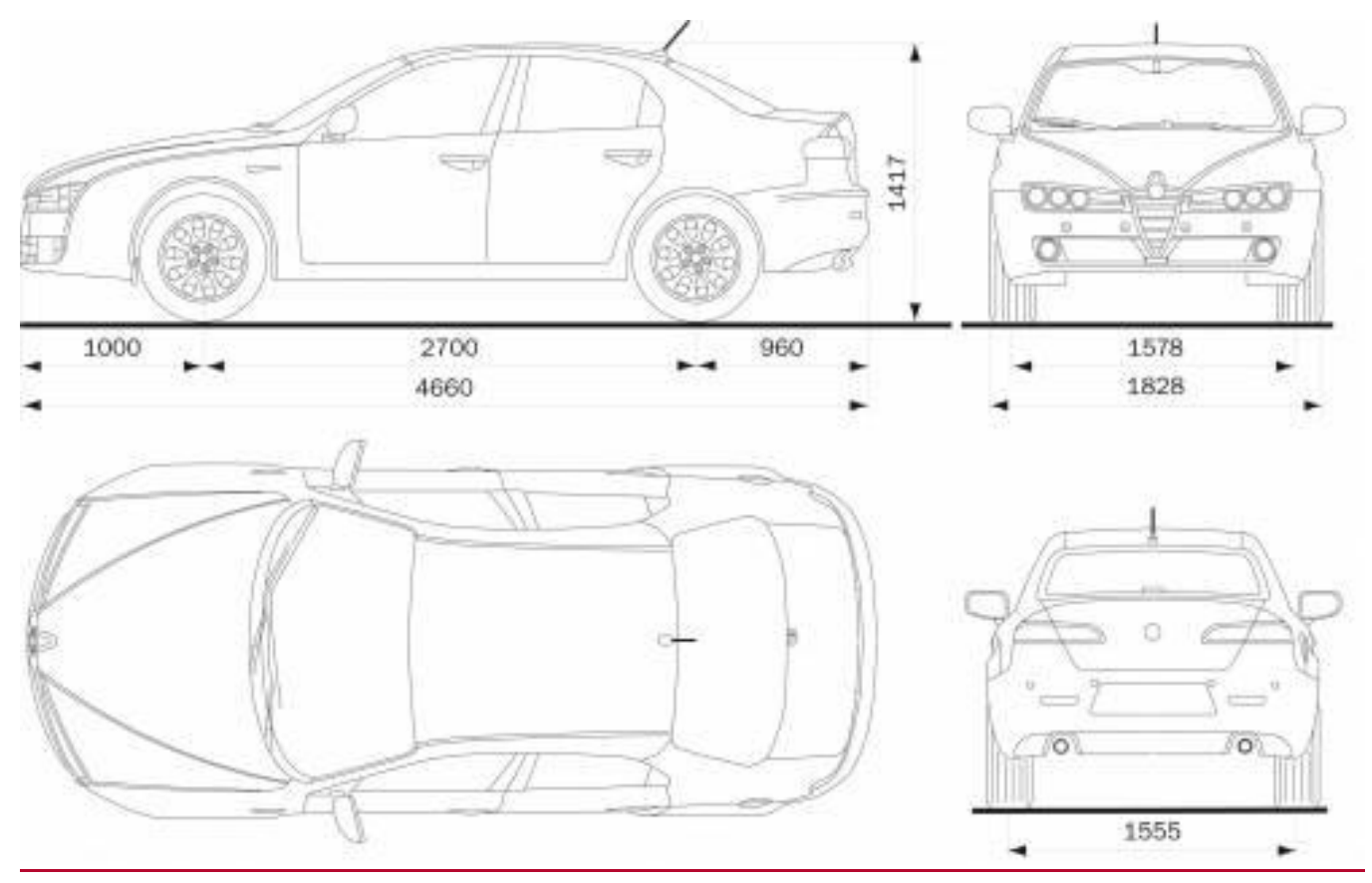

Figura 6 Principales Dimensiones Alfa Romeo 159

Fuente: https://8000vueltas.com/2010/09/15/citroen-gs-birotor

\section{Fiat 128}

- Comienzo Fabricación: 1971

- Término de Fabricación: 1978 
- Origen: Italia

- Denominación Original: Fiat 128

- Carrocería: Sedán autoportante

- Puertas: 4

- Motor: Fiat 4128 A 038 / 128 A $1.038(\mathrm{~L})$

- Ciclo: 4 tiempos, árbol de levas a la cabeza, válvulas a la cabeza

- Ubicación: Delantero transversal

- Capacidad Combustible (litros): 38

- Peso Vacío (Kg): 830

- Largo (mm): 3.884

- Ancho (mm): 1.590

- Alto (mm): 1.420

- Distancia entre Ejes (mm): 2.448

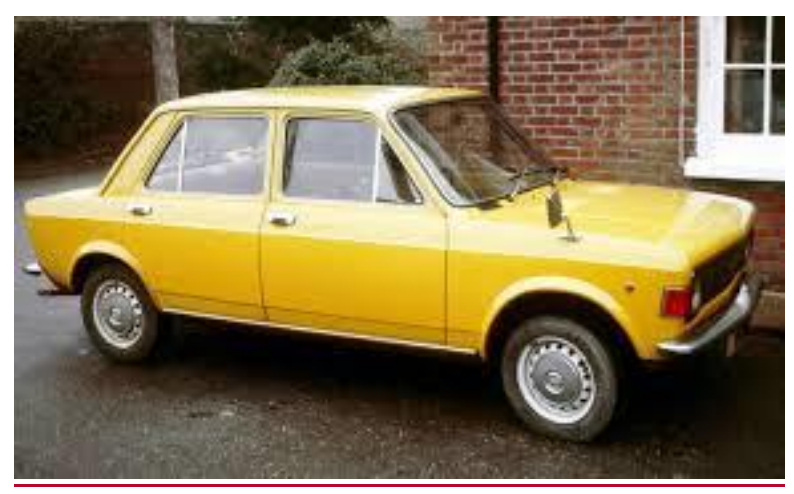

Figura 7 Fiat 128 Coeficiente de arrastre 0,48

Fuente: http://kiwix.demo.ideascube.org/wikipedia.es/A/Fiat_128.html 

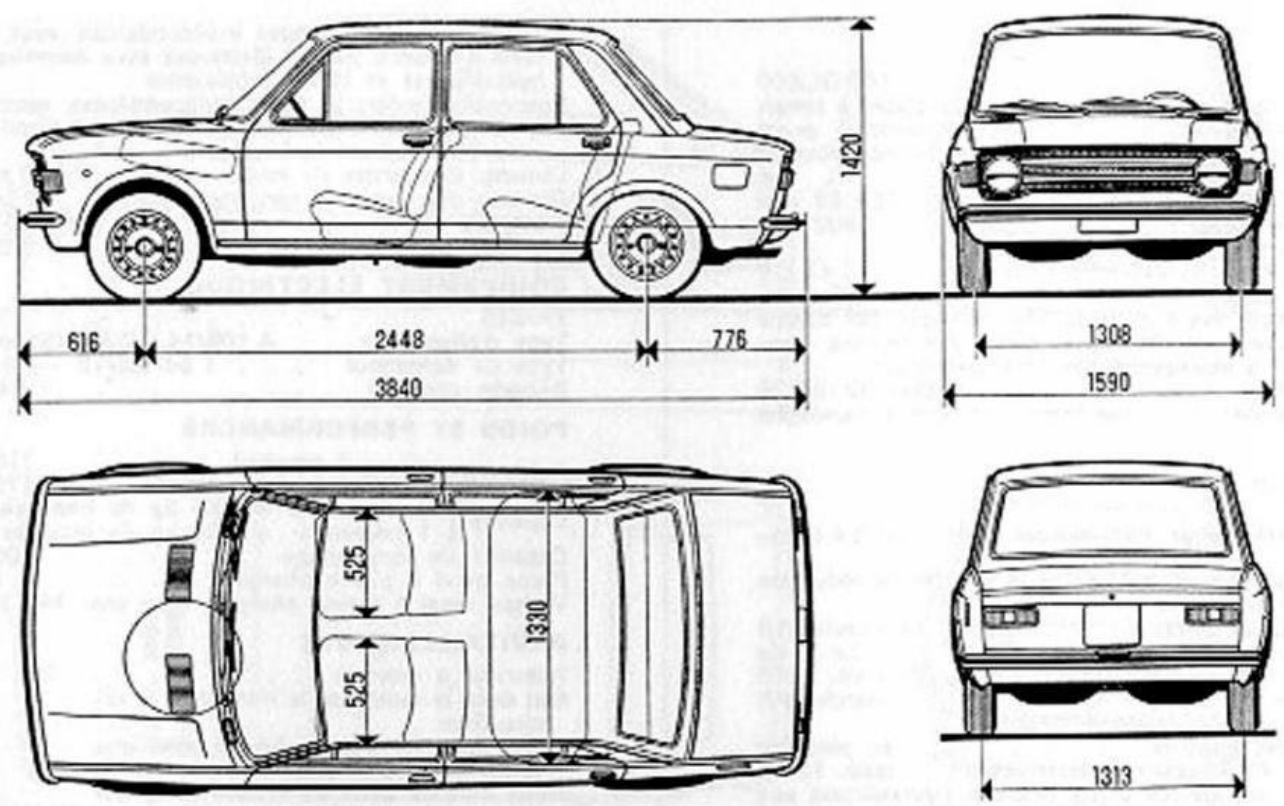

Volume du coffre: $370 \mathrm{dm}^{2}$.

La voiture pout être fournie en version 2 portes.

Figura 8 Principales Dimensiones Fiat 128

Fuente: http://kiwix.demo.ideascube.org/wikipedia.es/A/Fiat_128.html

\section{Cálculo del coeficiente de arrastre}

Como se había mencionado anteriormente, los cuerpos que están sometidos a la acción de un flujo de fluido generalmente agua o aire, es decir, flujos externos a través de ellos, experimentan una fuerza de resistencia a su paso, esta fuerza denominada fuerza de arrastre involucra un coeficiente $C_{d}$ (coeficiente de arrastre) en su ecuación matemática que a continuación se presenta y que está asociada con un modelo de superficie en particular.

$$
C_{d}=\frac{2 F_{d}}{\rho v^{2} A}
$$

Dónde:

$C_{d}:$ Coeficiente de arrastre

$F_{d}:$ Fuerza de arrastre

A: Área de referencia 
La densidad del fluido, la tomamos a una temperatura ambiente alrededor de $15^{\circ} \mathrm{C}$, la velocidad lo vamos a calcular en función del caudal, y se debe mencionar que en la práctica probamos con 7, 6, 5 y 4 1/s, después comprobaremos si efectivamente o no influye este factor.

El procedimiento experimental se describe a continuación:

1. Revisar el dinamómetro, de ser necesario volverlo a setear en la posición de cero, para obtener lecturas confiables.

2. Se ubica el cuerpo de estudio, tomando en cuenta que la cara frontal debe estar en oposición al movimiento.

3. Se pone en marcha el banco de flujo externo, comenzando por el valor más alto que puede proporcionar, el cual es 7 1/s, y progresivamente se disminuye este valor hasta llegar a un flujo de $21 / s$.

4. En cada variación de caudal se toma el valor indicado en el dinamómetro, mismo que refleja la fuerza de arrastre a la cual está sometido el cuerpo en estudio.

\section{Resultados}

Los resultados de la experimentación se presentan para cada uno de los modelos. En primera instancia se muestra los valores obtenidos en el laboratorio, luego se propone una ecuación a través de la curva obtenida de los datos, de tal manera que se llegue a identificar la independencia del número de Reynolds y con esto llegar a obtener el valor del coeficiente de arrastre que se podría utilizar de manera práctica.

\section{Vehículo Citroën Gs}

Tabla 1 Resultados experimentales Vehículo Citroën Gs

\begin{tabular}{|c|c|c|c|c|c|c|c|c|c|}
\hline \multicolumn{7}{|c|}{ Modelo $^{\circ} \mathbf{1}$ CITOEN GS } \\
\hline $\begin{array}{c}\text { Caudal } \\
{[\mathbf{l} / \mathbf{s}]}\end{array}$ & $\begin{array}{c}\text { Caudal } \\
{\left[\mathbf{m}^{\mathbf{3} / \mathbf{s}}\right]}\end{array}$ & $\boldsymbol{h}_{\boldsymbol{L}}[\mathbf{c m}] \boldsymbol{h}_{\boldsymbol{L}}[\mathbf{m}]$ & $\begin{array}{c}\text { Área } \\
{\left[\mathbf{m}^{\mathbf{2}}\right]}\end{array}$ & $\begin{array}{c}\text { Velocidad } \\
{[\mathbf{m} / \mathbf{s}]}\end{array}$ & $\boldsymbol{L}_{\boldsymbol{m}}[\mathbf{m}]$ Reynolds & $\mathbf{F}[\mathbf{N}]$ & $\mathbf{C d}$ \\
\hline 2 & 0,002 & 10,5 & 0,105 & 0,0105 & 0,19 & 0,074 & 12299 & 2,2 & 50,2 \\
\hline 3 & 0,003 & 12 & 0,12 & 0,012 & 0,25 & 0,074 & 16143 & 2,4 & 31,8 \\
\hline
\end{tabular}




\begin{tabular}{|c|c|c|c|c|c|c|c|c|c|}
4 & 0,004 & 13,3 & 0,133 & 0,0133 & 0,30 & 0,074 & 19420 & 2,65 & 24,2 \\
\hline 5 & 0,005 & 14,9 & 0,149 & 0,0149 & 0,34 & 0,074 & 21668 & 2,9 & 21,3 \\
\hline 6 & 0,006 & 15,2 & 0,152 & 0,0152 & 0,39 & 0,074 & 25488 & 3 & 15,9 \\
\hline
\end{tabular}

Fuente: Autores

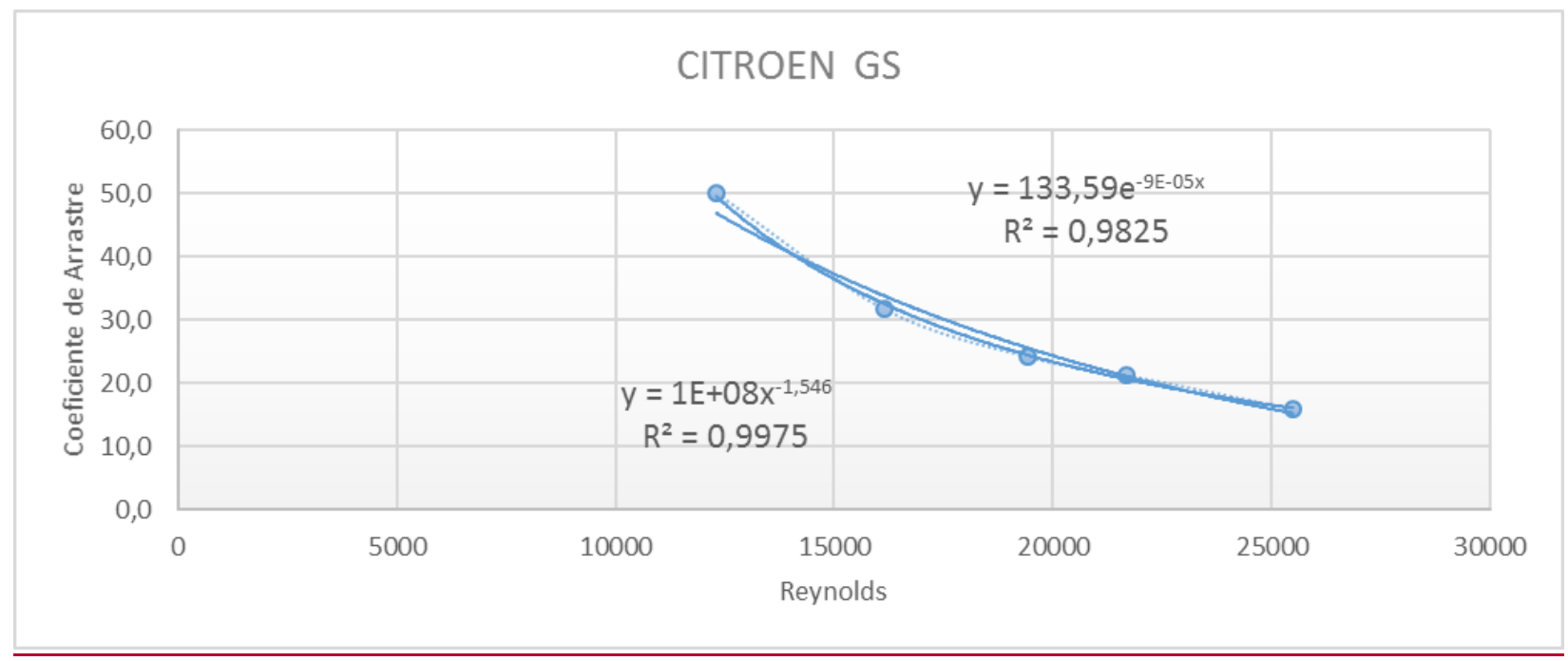

Figura 9 Relación entre el número de Reynolds y coeficiente de arrastre Vehículo Citroën Gs, curva experimental

\section{Fuente: Autor}

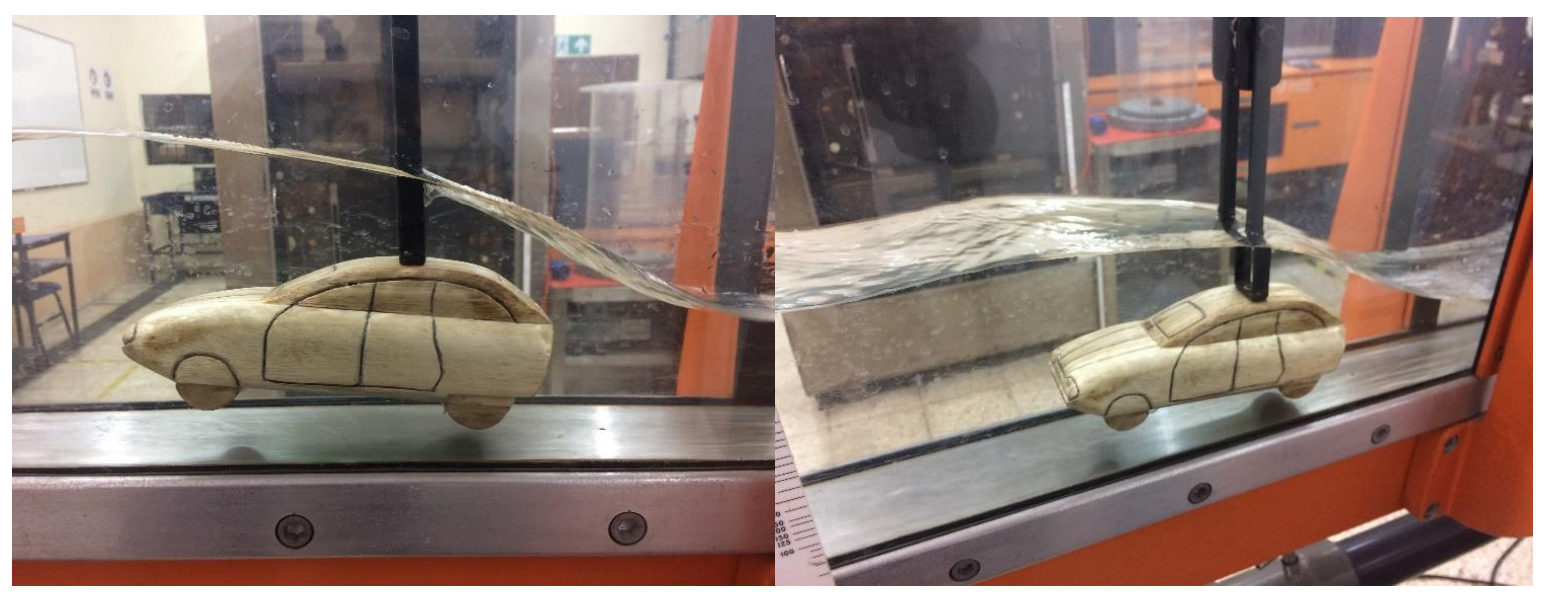

Figura 10 Experimentación para medición del coeficiente de arrastre Modelo 1

Fuente: Autores 


\section{Curva Modélo 3 CITROEN GS}

- Exponencial $\bullet$ Potencial

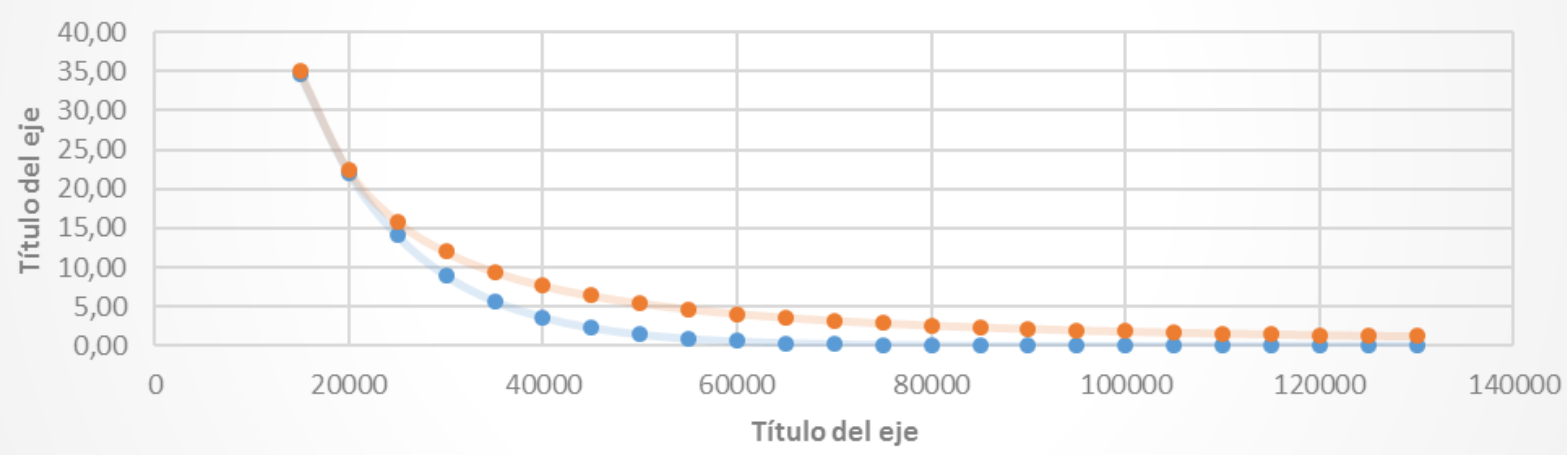

Figura 11 Relación entre el número de Reynolds y coeficiente de arrastre Vehículo Citroën Gs, curva propuesta

Fuente: Autores

El Valor del Número de Reynolds para en el que se aprecia un valor constante es a partir de los 72000 con un Coeficiente de 0,2

\section{Alfa Romeo 159}

Tabla 2 Resultados experimentales Vehículo Alfa Romeo 159

\begin{tabular}{|c|c|c|c|c|c|c|c|c|c|}
\hline \multicolumn{7}{|c|}{ Modelo $\mathbf{N}^{\circ}$ 2 ALFA ROMEO } \\
\hline $\begin{array}{c}\text { Caudal } \\
{[\mathbf{l} / \mathbf{s}]}\end{array}$ & $\begin{array}{c}\text { Caudal } \\
{\left[\mathbf{m}^{\mathbf{3}} / \mathbf{s}\right]}\end{array}$ & $\boldsymbol{h}_{\boldsymbol{L}}[\mathbf{c m}] \boldsymbol{h}_{\boldsymbol{L}}[\mathbf{m}]$ & $\begin{array}{c}\text { Área } \\
{\left[\mathbf{m}^{\mathbf{2}}\right]}\end{array}$ & $\begin{array}{c}\text { Velocidad } \\
{[\mathbf{m} / \mathbf{s}]}\end{array}$ & $\boldsymbol{L}_{\boldsymbol{m}}[\mathbf{m}] \mathbf{R e y n o l d s}$ & $\mathbf{F}[\mathbf{N}]$ & $\mathbf{C d}$ \\
\hline 2 & 0,002 & 10,7 & 0,107 & 0,0107 & 0,19 & 0,0776 & 12656 & 2,6 & 58,9 \\
\hline 3 & 0,003 & 12 & 0,12 & 0,012 & 0,25 & 0,0776 & 16928 & 2,8 & 35,5 \\
\hline 4 & 0,004 & 13,2 & 0,132 & 0,0132 & 0,30 & 0,0776 & 20519 & 3 & 25,9 \\
\hline 5 & 0,005 & 15 & 0,15 & 0,015 & 0,33 & 0,0776 & 22571 & 3,2 & 22,8 \\
\hline 6 & 0,006 & 15,5 & 0,155 & 0,0155 & 0,39 & 0,0776 & 26211 & 3,4 & 18,0 \\
\hline
\end{tabular}

Fuente: Autores 


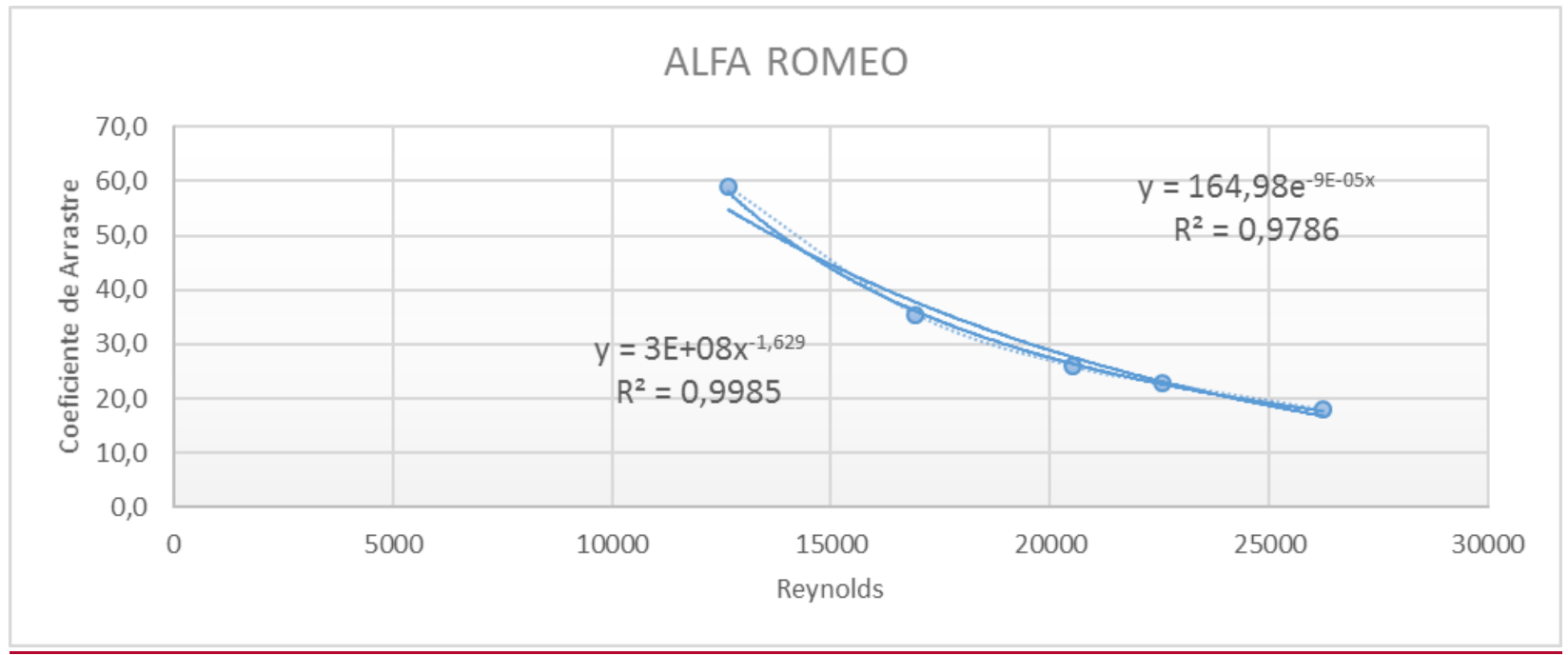

Figura 12 Relación entre el número de Reynolds y coeficiente de arrastre Vehículo Alfa Romeo 159, curva experimental

\section{Fuente: Autores}

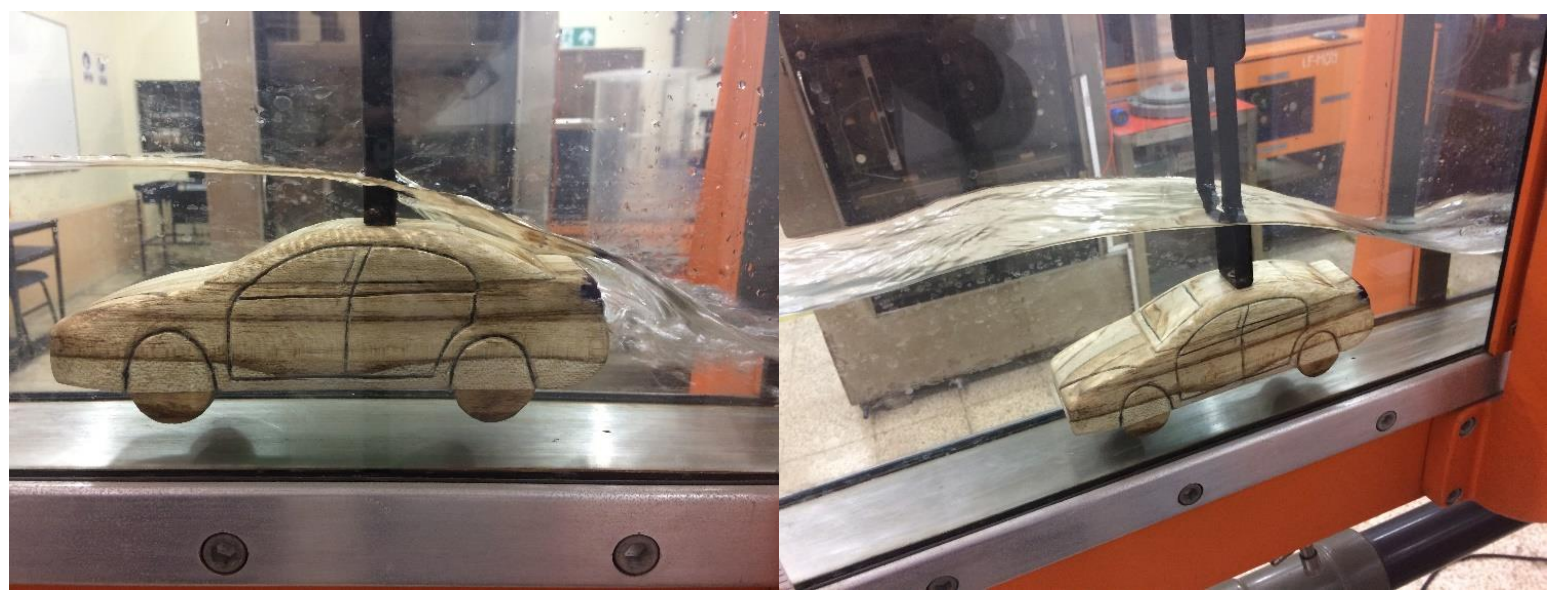

Figura 13 Experimentación para medición del coeficiente de arrastre Modelo 2

Fuente: Autores 


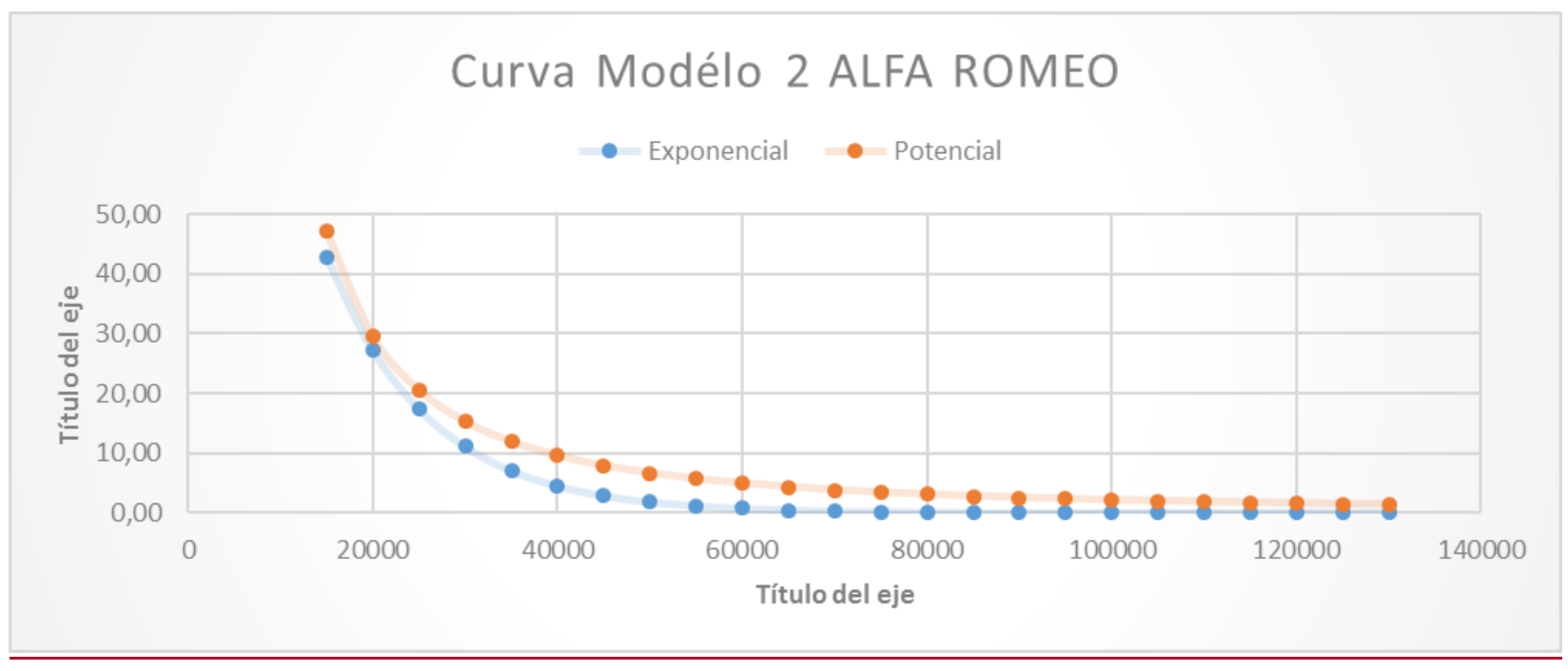

Figura 14 Relación entre el número de Reynolds y coeficiente de arrastre Vehículo Alfa Romeo 159, curva propuesta

\section{Fuente: Autores}

El Valor del Número de Reynolds para en el que se aprecia un valor constante es a partir de los 70000 con un Coeficiente de 0,25

\section{Fiat 128}

Tabla 3 Resultados experimentales Vehículo Fiat 128

\begin{tabular}{|c|c|c|c|c|c|c|c|c|c|}
\hline \multicolumn{10}{|c|}{ Modelo $^{\circ} \mathbf{1}$ FIAT 128 } \\
\hline $\begin{array}{c}\text { Caudal } \\
{[\mathbf{1} / \mathbf{s}]}\end{array}$ & $\begin{array}{c}\text { Caudal } \\
{\left[\mathbf{m}^{\mathbf{3}} / \mathbf{s}\right]}\end{array}$ & $\boldsymbol{h}_{\boldsymbol{L}}[\mathbf{c m}] \boldsymbol{h}_{\boldsymbol{L}}[\mathbf{m}]$ & $\begin{array}{c}\text { Área } \\
{\left[\mathbf{m}^{\mathbf{2}}\right]}\end{array}$ & $\begin{array}{c}\text { Velocidad } \\
{[\mathbf{m} / \mathbf{s}]}\end{array}$ & $\boldsymbol{L}_{\boldsymbol{m}}[\mathbf{m}]$ Reynolds & $\mathbf{F}[\mathbf{N}]$ & $\mathbf{C d}$ \\
\hline 2 & 0,002 & 9,5 & 0,095 & 0,0095 & 0,21 & 0,0663 & 12179 & 1,2 & 22,4 \\
\hline 3 & 0,003 & 11,1 & 0,111 & 0,0111 & 0,27 & 0,0663 & 15636 & 1,8 & 20,4 \\
\hline 4 & 0,004 & 12,5 & 0,125 & 0,0125 & 0,32 & 0,0663 & 18513 & 2,0 & 15,8 \\
\hline 5 & 0,005 & 13,5 & 0,135 & 0,0135 & 0,37 & 0,0663 & 21427 & 2,0 & 12,1 \\
\hline 6 & 0,006 & 14,5 & 0,145 & 0,0145 & 0,41 & 0,0663 & 23939 & 2,1 & 10,1 \\
\hline 7 & 0,007 & 15,2 & 0,152 & 0,0152 & 0,46 & 0,0663 & 26642 & 2,2 & 8,6 \\
\hline
\end{tabular}

\footnotetext{
Fuente: Autores
} 


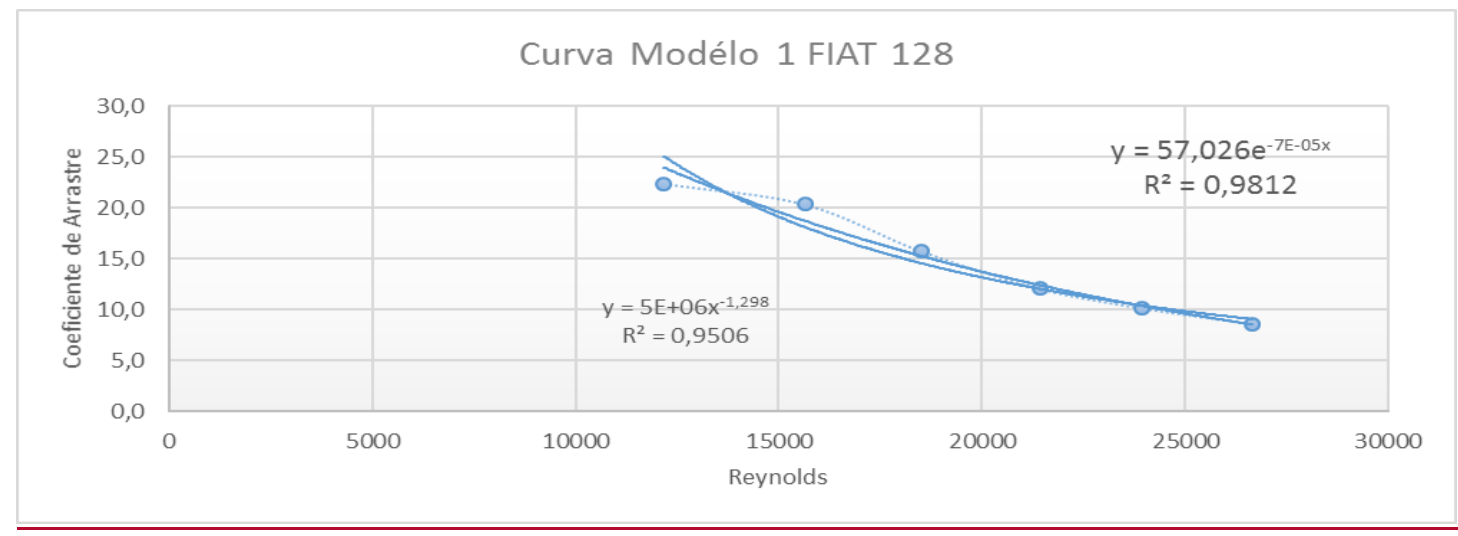

Figura 15 Relación entre el número de Reynolds y coeficiente de arrastre Vehículo Fiat 128, curva experimental

Fuente: Autores

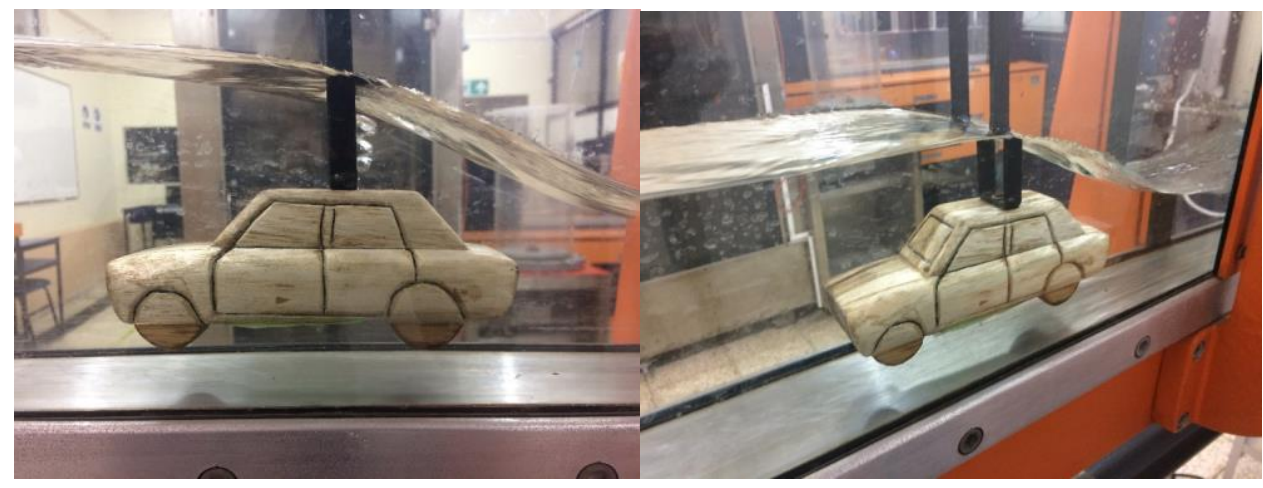

Figura 16 Experimentación para medición del coeficiente de arrastre Modelo 3

Fuente: Autores

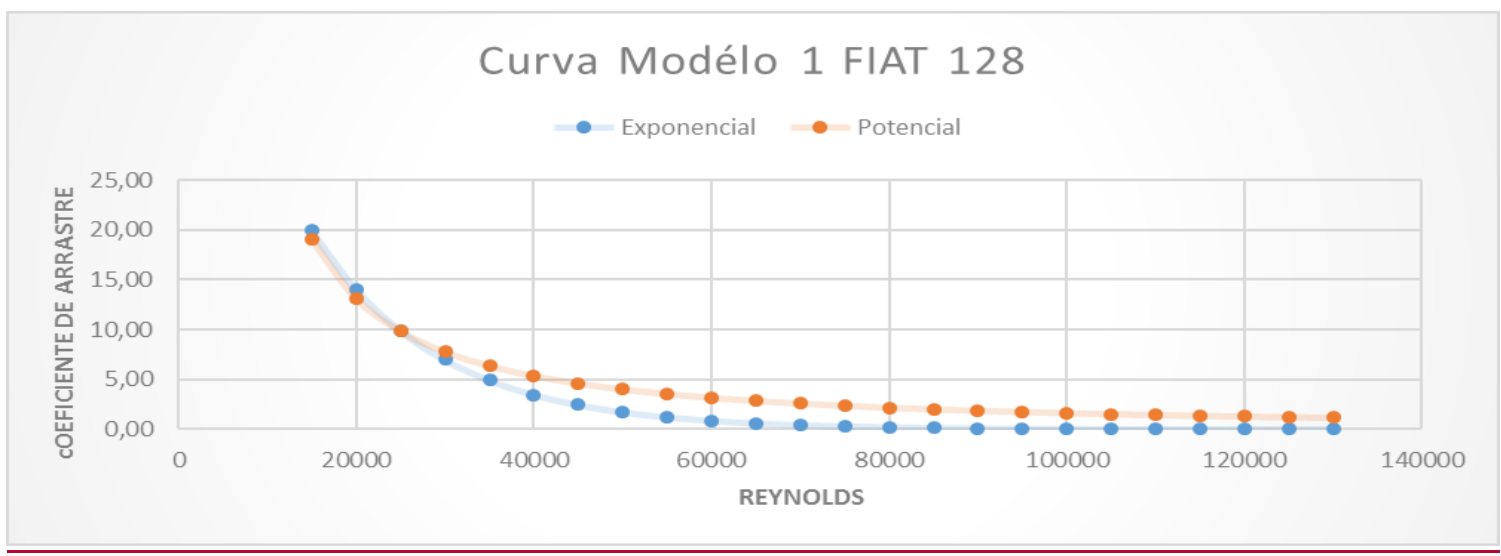

Figura 17 Relación entre el número de Reynolds y coeficiente de arrastre Vehículo Fiat 128, curva propuesta 
Fuente: Autores

El Valor del Número de Reynolds para en el que se aprecia un valor constante es a partir de los 72000 con un Coeficiente de 0,36 .

Finalmente, en la Tabla 4, se presenta un resumen de los valores de los coeficientes de arrastre de los modelos seleccionados, de esta manera se puede observar la diferencia que existe entre los valores teóricos y experimentales.

Tabla 4 Resumen de resultados Teóricos y experimentales

\begin{tabular}{|c|c|c|}
\hline MODELO & $\begin{array}{c}\text { COEFICIENTE DE } \\
\text { ARRASTRE TEORICO }\end{array}$ & $\begin{array}{c}\text { COEFICIENTE DE } \\
\text { ARRASTRE } \\
\text { EXPERIMENTAL }\end{array}$ \\
\hline Vehículo Citroën Gs & 0,3 & 0,2 \\
\hline Alfa Romeo 159 & 0,42 & 0,25 \\
\hline Fiat 128 & 0,48 & 0,36 \\
\hline
\end{tabular}

\section{Fuente: Autores}

\section{Conclusiones}

Para el cálculo de las áreas de los modelos a escala de los modelos de autos, es recomendable realizarlo en un software $\mathrm{CAD}$, por la complejidad en su forma, y proceder a calcular el área en dicho software, esto con el objetivo de llegar a tener mayor exactitud en el cálculo del coeficiente. Las velocidades necesarias para la experimentación dentro del canal son altas, si lo que se requiere es alcanzar la independencia del número de Reynolds, sin embargo, con el rango de velocidades que se cuenta es posible construir una curva Reynolds vs $\mathrm{Cd}$, de tal manera que esta nos indique en qué valor se llega a la esperada independencia. Al compararlos con los datos que provee el fabricante, teóricos, tienen un margen de error aproximadamente del 30\%; este porcentaje de error se puede mejorar al tener un modelo de mejor construcción en sus acabados y un cálculo de área más exacto, sin embargo, se está demostrando que las recomendaciones para estimar este tipo de coeficientes son muy válidas, y en laboratorio cualquier cuerpo que requiera ser estudiado en este contexto aerodinámico proveerá de resultados confiables. En el sentido comercial este valor del coeficiente de arrastre es un indicativo de que el vehículo que vaya a ser usado por un comprador, geométricamente posee buenas o malas características aerodinámicas, 
lo cual influirá directamente en el consumo de combustible del mismo. Si lo que se pretende es llegar a ser muy cuidadoso con los factores que influye en la eficiencia del vehículo, la aerodinámica del mismo es un parámetro muy importante.

\section{Referencias Bibliográficas}

1. Campuzano, Eliezer. Coeficiente de arrastre. [En línea] 2009. [Citado el: 06 de 05 de 2018.] https://sites.google.com/site/0902eliezerc/coeficiente-de-arrastre.

2. Modeling drag force acting on the individual particles in low Reynolds number flow. Lee, SangHwan y Kim, JuHyeon. s.1.: Elsevier, 14 de abril de 2014, Powder Technology, págs. 22-32.

3. Drag force in wind tunnels: A new method. Olivei, P.M.C, Girardi, D y Souza, P.V.S. s.l.: Elsevier, Physica A, Vol. 467, págs. 120-128.

4. Norplan Engineering. Technical courses. [En línea] [Citado el: 06 de 05 de 2018.] http://www.technicalcourses.net/portal/es/blog/blog_entrada.php?entrada_id=89..

5. Cengle, Yunus y Cimbala, Jhon. Mecánica de Fluidos. México D.F.: McGraw Hill, 2006. ISBN 970-10-5612-4.

6. Happel, H y Brenner, J. Low Reynolds Number Hydrodynamics:With Special Applications to particulate media. Miami: The Hague, 1983. Vol. 1.

7. Drag and torque on clusters of N-arbitrary spheres at low Reynolds number. Filippov, A. s.l.: Elsevier, september de 1 de 2000, Vol. 229, págs. 184-195.

8. Simulation of the hydrodynamic drag of aggregated particles. Binder, Christian, y otros. 1, s.1.: Elsevier, 1 de septiembre de 2006, Journal of Colloid and Interface Science, Vol. 301, págs. 155 167.

9. Munson, B y Young, D. Fundamentals of fluid mechanics. Miami: Oceanography Literature review, 1990. 0967-0653. 